\title{
USING MANUAL MEASUREMENTS ON EVENT RECORDER VIDEO AND IMAGE PROCESSING ALGORITHMS TO EXTRACT OPTICAL PARAMETERS AND RANGE
}

\author{
Jonas Bärgman $^{1}$, Julia Werneke ${ }^{1}$, Christian-Nils Boda ${ }^{1}$, Johan Engström ${ }^{2}$ \& Kip Smith ${ }^{3}$ \\ ${ }^{1}$ Chalmers University of Technology, Division of Vehicle Safety, Göteborg, Sweden \\ ${ }^{2}$ AB Volvo, Göteborg, Sweden \\ ${ }^{3}$ Naval Postgraduate School, Monterey, California, USA \\ Email: jonas.bargman@chalmers.se
}

\begin{abstract}
Summary: Vehicle kinematics and optical parameters such as optical angle, optical expansion rate, and tau are thought to underlie drivers' ability to avoid and handle critical traffic situations. Analyses of these parameters in naturalistic driving data with video, such as commercial event recordings of near-crashes and crashes, can provide insight into driver behavior in critical traffic situations. This paper describes a pair of methods, one for the range to a lead vehicle and one for its optical angle, that are derived from image processing mathematics and that provide driver behavior researchers with a relatively simple way to extract optical parameters from video-based naturalistic data when automatic image processing is not possible. The methods begin with manual measurements of the size of other road users on a video on a screen. To develop the methods, 20 participants manually measured the width of a lead vehicle on 14 images where the lead vehicle was placed at different distances from the camera. An on-market DriveCam Event Recorder was used to capture these images. A linear model that corrects distortion and modeling optics was developed to transform the on-screen measurements distance (range) to and optical angle of the vehicle. The width of the confidence interval for predicted range is less than $0.1 \mathrm{~m}$ when the actual distance is less than $10 \mathrm{~m}$ and the lead-vehicle width estimate is correct. The methods enable driver behavior researchers to easily and accurately estimate useful kinematic and optical parameters from videos (e.g., of crashes and nearcrashes) in event-based naturalistic driving data.
\end{abstract}

\section{INTRODUCTION}

In the development of in-vehicle active safety systems, it is important to understand both the kinematics of the vehicles involved and drivers' decision making. The former include the use of parameters such as range, range-rate, and time headway. Research on driver decision making has focused on how humans make decisions related to time-to-contact in different contexts. Much of this research has adhered to optical flow theory and the tradition of ecological psychology developed by Gibson to focus on how drivers estimate time-to-contact (Gibson 1958; Gibson \& Crooks, 1938). Lee (1976) suggested that the decision to initiate braking is determined by an optically specified time-to-collision called tau $(\tau)$. Here, tau is defined as the ratio of the optical angle of an object $(\theta)$ and its time derivative $(\dot{\theta})$. In the context of driving, several authors have argued for tau as a basis for the decision to brake (Schiff \& Detwiler, 1979; Yilmaz \& Warren, 1995). In contrast, others have shown that tau seems to be only part of the information subjects use to estimate time-to-collision (DeLucia, 2004; Hancock \& Manser, 1997). Common across the driving literature is the claim that decisions to initiate control actions, such as braking, are based on optical parameters which drivers pick-up from the environment while driving. 
The main methods employed in the time-to-collision research have been experimental. Many authors use driving simulators (Gray et al., 1999), scenario visualization by, e.g., video sequences (Hancock \& Manser, 1997), or test-track driving (Kiefer et al., 2006). With the recent advent of naturalistic driving studies (e.g., Dingus et al., 2006), a new method has emerged to collect data about other road users in normal everyday driving as well as in critical traffic situations. The data can be used to investigate e.g. the correlation between optical parameters and drivers' behavior in both normal and critical situations. This unobtrusive collection of data provides a relatively detailed record of the driver and vehicle in relation to other road users. For example, installing a radar device on a study vehicle provides direct information about the kinematic parameters range and range-rate to a lead vehicle, and optical parameters such as optical angle, optical expansion rate, and tau can be derived. In contrast, in naturalistic driving studies that do not include automatic means of getting range and range-rate, this information is available only indirectly from post-hoc examination of video sequences of the roadway ahead (including the leading vehicle). To automate the parameter extraction, image processing software can often be used. However, new sources of naturalistic data are emerging where researchers may not have the possibility to access the videos but are able to get the videos annotated.

This paper describes two methods for extracting the parameters range, optical angle, optical expansion rate, and tau from video collected as part of naturalistic driving studies, using DriveCam (2012) data. Driver behavior researchers can use the methods if automatic extraction of the parameters from video is not possible, e.g. not available to the researchers, or if they are not image processing experts.

\section{METHOD}

The methods were developed using established image-processing algorithms for rectifying video and estimating the range to a lead vehicle and the optical angle, optical expansion rate, and tau of this vehicle from the driver's perspective. This section describes the data source used, the measurements performed, and example calculations of range and optical parameters.

\section{Data Source}

The Event Recorder system used in this study was supplied by DriveCam (2012). DriveCam is a commercial company providing a service primarily to commercial fleets that seek to reduce collision-related expenses. The event-based video recorder monitors both the driver and the roadway ahead. Events are identified by kinematic triggers (e.g. acceleration thresholds being passed) or by the driver pressing an event button on the Event Recorder. The DriveCam Event Recorder records video at $4 \mathrm{~Hz}$, and stores data from $8 \mathrm{~s}$ before until $4 \mathrm{~s}$ after the trigger. The event data, including video, GPS, and acceleration, is uploaded to the DriveCam Data Center.

DriveCam personnel manually review the video and analyze the events, highlighting causes, and score the safety concerns. Feedback is then given to the customers. In this paper, the recorded event videos are treated as a rich source of data for traffic accident research. 


\section{Measurements of Lead Vehicle Width on Video}

It is helpful but not necessary to know the actual width of the lead vehicle to calculate optical parameters from video. However, it is essential to have complete and accurate information about the camera optics. The width of the vehicle can be known by identifying the exact vehicle model or it may be estimated based on vehicle type.

The following steps were performed to collect data for method development: First, a lead vehicle with a known width $\left(1.56 \mathrm{~m}, \mathrm{~S}_{\mathrm{t}}\right)$ was placed at 14 different ranges from a fixed DriveCam Event Recorder. The ranges were 1, 2, 3, 5, 7, 10, 15, 20, 30, 40, 50, 75, 100, and 125m. Thus, smaller steps were chosen for shorter ranges than for longer ranges, since one of the foci in this paper is to derive optical parameters, which are more relevant at short ranges. A measuring tape was used for the measurements and the estimated error was $\pm 0.1 \mathrm{~m}$ at ranges greater than $10 \mathrm{~m}$, and for shorter ranges less than $\pm 0.05 \mathrm{~m}$. The vehicle was facing away from the camera, simulating a carfollowing scenario. The range measurements were made from the Event Recorder to the rear bumper of the vehicle. Second, for each range an event was manually triggered on the Event Recorder using the manual trigger button. Finally, one frame for each range was extracted.

A total of 20 participants (10 male, 10 female), recruited at SAFER in Göteborg, with a mean age of 33.6 years ( $\mathrm{SD}=9.2$ years), manually measured the width of the lead vehicle with a millimeter scaled ruler, on each of the 14 images. These measurements were used as input to the calculations of range and optical parameters. Two out of the 280 measures (14 images $\mathrm{x}$ 20 participants) were excluded since they were too small to be correct. The width was measured on a standard 24 inch computer screen with the image at maximum possible size on the screen producing a total width of the visible video of $449 \mathrm{~mm}\left(\mathrm{~W}_{\mathrm{S}}\right)$. The instructions to the participants were: "Please measure the width as well as possible between the outer edges of the two rearlights. If the edges are not clear, please measure where your best estimate is of the position of these edges. You may use half millimeter steps if you need.” Each participant measured every image once. The order of the images was randomized. The overall experiment took approximately 10 minutes to complete, of which approximately 3 minutes where measurements.

\section{Calculations of Range and Optical Parameters from Measurements}

Rectification equations are available to compensate for optical distortion in an image (Heikkilä \& Silvén, 1997). The pixel coordinates on the image of the lead vehicle's left and right side rearlight measurements were rectified assuming the measurements being in the center of the screen. The rectification was done in two steps. First, the camera's lens's focal length ( $\mathrm{f}=545.9$ pixels) and parameters for rectifying the image distortion were extracted using the Camera Calibration Toolbox for Matlab (Bouguet, 2010). Second, these parameters were used with algorithms from the same toolbox together with linear regression to produce a linear model of the rectification specifically for the measurements of a lead vehicle on the DriveCam Event Recorder video.

A vector with artificially created vehicle widths $\left(S_{\text {dist }}\right)$ on the screen from $5 \mathrm{~mm}$ (corresponding to approximately $100 \mathrm{~m}$ range) to $400 \mathrm{~mm}$ ( $<1 \mathrm{~m}$ range) in steps of $1 \mathrm{~mm}$ was rectified using algorithms from the Camera Calibration Toolbox for Matlab (Bouguet, 2010). The result was a 
vector with rectified widths $\left(\mathrm{S}_{\text {rect }}\right)$. A third order least squares linear regression was applied to predictor $S_{\text {dist }}$, finding a best fit polynomial to $S_{\text {rect. }}$ To allow this equation to be used with other screen sizes, screen scaling was also introduced where $\mathrm{W}_{\mathrm{NS}}$ is the new screen size in millimeters. The resulting polynomial is shown in Equation 1 and has a mean error of $0.03 \mathrm{~mm}(\mathrm{SD}=0.5 \mathrm{~mm})$ across the 396 data points.

$$
S_{\text {rect }}=\left(0.37+1.0127 \cdot S_{\text {dist }} \cdot \frac{W_{S}}{W_{N S}}-0.000203 \cdot\left(S_{\text {dist }} \cdot \frac{W_{S}}{W_{N S}}\right)^{2}+0.00000151 \cdot\left(S_{\text {dist }} \cdot \frac{W_{S}}{W_{N S}}\right)^{3}\right) \cdot \frac{W_{N S}}{W_{S}}(1)
$$

The vehicle widths measured by the participants were rectified using Equation 1 to create a second least squares linear regression model, Equation 2, that predicts the range to the lead vehicle. This model is a transfer function between the rectified on-screen measured vehicle widths $1 / S_{\text {rect }}$ and actual range to the lead vehicle $R_{\text {real }}$. Use of the inverse of $S_{\text {rect }}$ is prescribed by standard optics equations. Note that only measurements from $1 \mathrm{~m}-10 \mathrm{~m}$ were used in the model fitting because range estimates are likely to be better at shorter ranges. To allow for use of this equation also with other vehicle widths, scaling is introduced where $\mathrm{S}_{\mathrm{NCreal}}$ is the new vehicle width in meters, and $S_{t}$ the width of the vehicle used in the method development $(1.56 \mathrm{~m})$. Equation 2 shows the result from the linear regression with the scaling.

$$
R_{\text {real }}=-0.278+\frac{559.27}{S_{\text {rect }}} \cdot \frac{S_{t}}{S_{N C r e a l}}
$$

The optical angle $(\theta)$ was calculated using Equation 3, where $S_{\text {rect }}$ is the rectified manual onscreen measurements, $W_{\text {NSrect }}$ is the maximum screen width in millimeters after rectification (equation 1 with $S_{\text {dist }}=W_{N S}$, here $W_{N S}=449$ ), $f$ is the focal length of the camera lens (here $\mathrm{f}=545.9$ ), and $\mathrm{W}_{\text {pix }}$ is the width of the video in pixels (here $\mathrm{W}_{\text {pix }}=655$ ). The optical expansion rate ( $\dot{\theta}$, the time derivative of $\theta$ ) and tau ( $\tau$, the ratio of $\theta$ to $\dot{\theta}$ ) of the lead vehicle can be easily calculated. Since static images cannot be used for calculation of $\dot{\theta}$, a second data collection was performed with the vehicle in motion. The lead vehicle was, instead of being static, accelerating away from the Event Recorder as it was recording video. To simulate a closing-lead-vehicle situation, the time vector was reversed.

$$
\theta=2 \cdot \arctan \left(\frac{\left(\frac{S_{\text {rect }}}{2}\right)}{\frac{\mathrm{f} \cdot W_{\text {NSrect }}}{W_{\text {pix }}}}\right)
$$

\section{Summary of the Methods}

The methods to calculate range and optical parameters related to a lead vehicle from a DriveCam Event Recorder video have four and five steps, respectively. To calculate range, first find or estimate the actual width of the vehicle in the image. Second, measure with a ruler the width of the vehicle in the video when displayed on an ordinary computer monitor. Third, measure the width of the total visible area of the event video. Fourth, apply Equations 1 and 2 consecutively, with the actual lead-vehicle width and video width. This transforms the on-screen measurements 
to the range to the vehicle. For the calculation of optical angle there are five steps. First, measure with a ruler the width of the vehicle in the video when displayed on an ordinary computer monitor. Second, measure the total visible width of the event video. Third, use the width from step 2 to rectify the measurement of total visible area using Equation 1. Fourth, rectify the onscreen measurements with equation 1 , and finally calculate the optical parameters using Equations 3 and its time derivative as well as $\tau$ as the ratio of $\theta$ to $\dot{\theta}$.

\section{RESULTS}

This section discusses the accuracy of the methods and some sources of error. As shown in Figure 1, the method accurately predicts ranges less than $10 \mathrm{~m}$. For ranges between $10 \mathrm{~m}$ and $40 \mathrm{~m}$ - car-following - the error is less than $5 \%$ if the lead vehicle width estimation is accurate. The method should be used with care for ranges over $40 \mathrm{~m}$ and sensitivity analysis should be made. For optical parameter calculation, care needs to be taken when estimating the time derivatives based on manual measurements. When used on real event data, it is often advantageous to use time-domain filters to smooth ranges and optical angle estimates based on the on-screen width measurements. Further, care has to be taken to estimate the vehicle width correctly when it is not known. Misestimating the actual width of the lead-vehicle will have a direct impact on the error in the calculation of range. Finally, lead vehicle occlusions or situations where the edges of the lead vehicle are outside of the image will create false parameter estimates.

Figure 1 shows the range prediction errors when the 278 measurements were compared with the model predictions using Equation 2 with $S_{\text {rect }}$ substituted from Equation 1. The mean error across the six ranges $\leq 10 \mathrm{~m}$ was $-0.37 \mathrm{~mm}(\mathrm{SD}=55.00 \mathrm{~mm})$. For the ranges $15-40 \mathrm{~m}$ the mean error was $-0.90 \mathrm{~m}(\mathrm{SD}=1.74 \mathrm{~m})$. Finally, for ranges from $50-125 \mathrm{~m}$ the mean error was $-3.27 \mathrm{~m}$ $(\mathrm{SD}=11.66 \mathrm{~m})$.
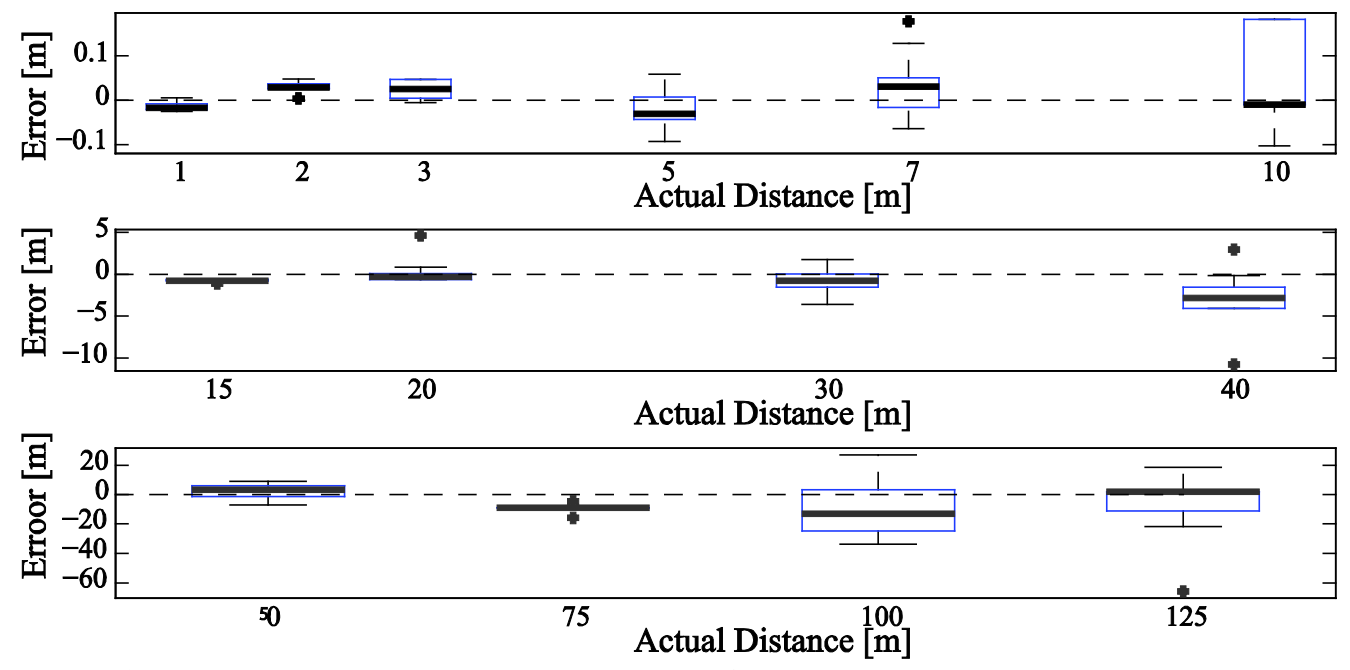

Figure 1. Top, middle, and bottom panels show the error for measured ranges $1-10 \mathrm{~m}, 15-40 \mathrm{~m}$, and $50-125 \mathrm{~m}$, respectively. The thick solid black horizontal line is the mean and the center of the boxplot is the median

Using the actual vehicle width in this paper it is possible to calculate the effect of misestimating the actual vehicle width on the range estimate. As an example, given a $\pm 0.1 \mathrm{~m}$ misestimation of 
the width and using $\mathrm{S}_{\mathrm{NCreal}}=1.56 \mathrm{~m}$ in $\mathrm{R}_{\text {error }}=1-\left(\mathrm{S}_{\mathrm{NCreal}} \pm 0.1\right) / \mathrm{S}_{\mathrm{NCreal}}$, the resulting error in the estimate of range is $\pm 6.4 \%$ of the real distance.

The left pane in Figure 2 shows $\theta, \dot{\theta}$ and, inverse $\tau$, based on calculations using Equation 3 for the simulated frame-by-frame images of closing-lead-vehicle data at $4 \mathrm{~Hz}$. The right pane in Figure 2 shows the effect of 1 and $2 \mathrm{~mm}$ errors in the width measure on the calculated values of optical angle. Errors in $\tau$ are magnified by differentiation in the calculation of $\dot{\theta}$.
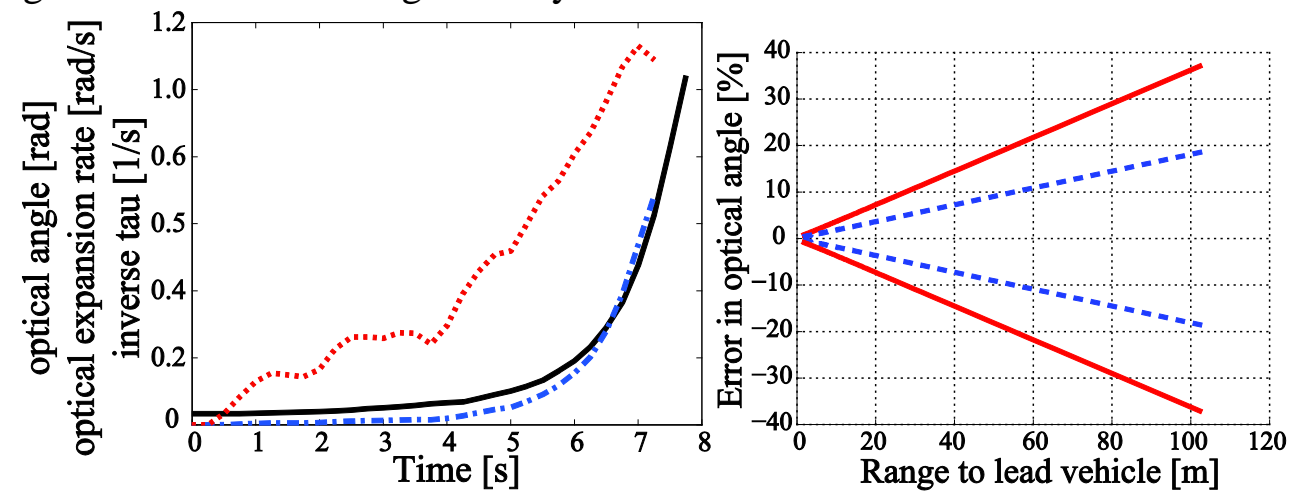

Figure 2. Left pane: optical angle (solid, black), optical expansion rate (dot-dashed, blue) and inverse tau (dotted, red) as a function to time, calculated from data from a lead-vehicle scenario example.

Right pane: error in optical angle corresponding to $\pm 1 \mathrm{~mm}( \pm 1.45$ pixels, dashed blue) and

\section{DISCUSSION}

$\pm 2 \mathrm{~mm}$ ( \pm 2.9 pixels, red) errors in on-screen measurement

This paper describes two methods derived from established algorithms for estimating ranges from video. The methods can be applied to event-based video data recordings like those obtained in commercial naturalistic driving data. The range and optical parameters could be used by researchers to better understand when and why drivers react the way they do in critical situations as well as for other driver behavior research. The range information can be used to derive other kinematic parameters such as range-rate, time-to-collision and time- and distance headway.

The methods were developed for extracting optical parameters and kinematics from video from DriveCam Event Recorders. Only the constants in Equations 1 and 2 are likely to change if another video system is used. Equation 2 can be used without rectifying the measured widths through Equation 1, but then the parameter estimates will have a considerable error at closer ranges. Further, if image processing is available, only the lead vehicle width in pixels is needed to extract optical parameters from a slightly modified Equation 3.

In conclusion, the methods developed here can be used to extract range information to a lead vehicle and corresponding optical parameters from video data obtained in commercial event based naturalistic driving data, especially when radar is not available. The use of video based observation throughout the pre-crash phase is likely to provide more accurate estimates of the evolving of the event than the traditional methods that include retrospective reconstruction from on-scene investigations. The resulting information and derivatives thereof is relevant for driver behavior research in critical traffic situations. 


\section{ACKNOWLEDGMENTS}

The methods were developed within the ANNEXT project (ANalysis of Naturalistic EXTernal datasets) sponsored by VINNOVA. The work was performed within SAFER Vehicle and Traffic Safety Centre, at Chalmers University of Technology. We would like to thank DriveCam for providing us with one of their Event Recorders and Dr. Marco Dozza for his valuable support.

\section{REFERENCES}

Bouguet, J.-Y. (2010). Camera Calibration Toolbox for Matlab. Retrieved November 19th, 2012, from http://www.vision.caltech.edu/bouguetj/calib_doc/index.html

DeLucia, P. R. (2004). Multiple sources of information influence Time-to-Contact judgements: Do heuristics accomodate limits in sensory and cognitive processes? In H. Hecht \& G. J. P. Savelsbergh (Eds.), Time-to-contact (Vol. 135, pp. 241-285). Amsterdam: Elsevier.

Dingus, T. A., Klauer, S. G., Neale, V. L., Petersen, A., Lee, S. E., Sudweeks, ... Jermeland, J. (2006). The 100-car naturalistic driving study, Phase II - results of the 100-car field experiment. Washington, DC: National Highway Traffic Safety Administration.

DriveCam. (2012). DriveCam The Driver Science Company. Retrieved November 19th, 2012, from www.drivecam.com

Gibson, J. J. (1979). The ecological approach to visual perception. Boston: Houghton Mifflin.

Gibson, J. J., \& Crooks, L. E. (1938). A theoretical field-analysis of automobile driving. The American Journal of Psychology, 51(3), 453-471.

Gray, R., Regan, D. M., \& Supplement, E. A. (1999). Adaptation to expansion and perceived time to collision: psychophysical and driving-simulator observations. Perception, ECVP Abstract Supplement 28.

Hancock, P. A., \& Manser, M. P. (1997). Time-to-contact: More than tau alone. Ecological Psychology, 9(4), 265-297.

Heikkilä, J., \& Silvén, O. (1997). A Four-step Camera Calibration Procedure with Implicit Image Correction. Paper presented at the IEEE Computer Society Conference on Computer Vision and Pattern Recognition, San Juan, Puerto Rico.

Kiefer R.J, Flannagan C.A, \& C.J., J. (2006). Time-to-Collision Judgments Under Realistic Driving Conditions. Journal of the Human Factors and Ergonomics Society, 48, 334-345.

Lee, D. N. (1976). A theory of visual control of braking based on information about time-tocollision. Perception, 5, 437-459.

Schiff, W., \& Detwiler, M. (1979). Information used in judging impending collision. Perception, 8, 647-658.

TRB. (2012). Frequently Asked Questions about the SHRP 2 Naturalistic Driving Study. Retrieved Nov 21, 2012, from http://onlinepubs.trb.org/onlinepubs/shrp2/NDSFAQ.pdf

Yilmaz, E. H., \& Warren, W. H. (1995). Visual control of braking: A test of the tau hypothesis. Journal of Experimental Psychology, 21(5), 996-1014. 